\title{
Le prix des paysages périurbains
}

Price of Periurban Landscapes

Jean Cavailhès, Thierry Brossard, Mohamed Hilal, Daniel Joly, PierreFrançois Tourneux, Céline Tritz et Pierre Wavresky

\section{(2) OpenEdition}

\section{Journals}

Édition électronique

URL : http://journals.openedition.org/economierurale/2003

DOI : $10.4000 /$ economierurale.2003

ISSN : 2105-2581

Éditeur

Société Française d'Économie Rurale (SFER)

Édition imprimée

Date de publication : 6 mai 2007

Pagination : 71-84

ISSN : 0013-0559

Référence électronique

Jean Cavailhès, Thierry Brossard, Mohamed Hilal, Daniel Joly, Pierre-François Tourneux, Céline Tritz et Pierre Wavresky, «Le prix des paysages périurbains », Économie rurale [En ligne], 297-298 | janvier-avril 2007, mis en ligne le 01 mars 2009, consulté le 19 avril 2019. URL : http://journals.openedition.org/ economierurale/2003; DOI : 10.4000/economierurale.2003 


\section{Le prix des paysages périurbains}

Jean CAVAILHÈS (a), Thierry BROSSARD (b), Mohamed HILAL (a), Daniel JOLY (b), Pierre-François TOURNEUX (b), Céline TRITZ (b), Pierre WAVRESKY (a)

(a) INRA-CESAER, Centre d'économie et de sociologie rurales appliquées à l'agriculture et aux espaces ruraux, Dijon • (b) CNRS-ThéMA, Théoriser et modéliser pour aménager, Besançon

\section{Introduction}

L a plupart des pays développés connaissent un mouvement d'extension des villes vers les campagnes environnantes (Caruso, 2002). En France, de 1990 à 1999, l'extension des espaces périurbains a été remarquable : leur nombre d'habitants s'est accru de 3,5 millions pour atteindre 12,3 millions tandis que leur surface augmentait de $50 \%$. Avec 6 millions d'hectares conquis, leur emprise concerne 15000 communes et représente $33 \%$ du territoire national.

Des tendances comparables s'observant dans presque tous les pays riches, la question de leurs déterminants s'inscrit sur l'agenda des scientifiques. La plupart des auteurs soulignent aujourd'hui le rôle des aménités « vertes » et du cadre de vie « rural » pour expliquer l'attrait des espaces périurbains. Par exemple, pour la France, Le Jeannic (1997), parle du goût pour «vivre dans un cadre naturel loin de l'agitation des villes, dans des maisons spacieuses et pourvues de jardins, tout en conservant la source de rémunération qu'est l'emploi en ville ».

En effet, l'économie urbaine (Brueckner et al, 1999 ; Fujita et Thisse, 2002) montre que le choix d'une localisation résidentielle résulte d'un arbitrage entre l'accessibilité à la ville (emplois, services, biens publics), le coût du logement (qui diminue avec l'éloignement) et la présence de nuisances ou d'aménités, parmi lesquelles les paysages.

Les agriculteurs en particulier, même s'ils sont critiqués pour leurs nuisances (odeurs, pollutions, bâtiments disgracieux, etc.), entretiennent et gèrent l'espace, qui est ouvert à la promenade, offre des paysages agréables et donne à voir une image de nature ou de ruralité qui plaît aux Français.
Ils produisent une externalité dont jouissent les habitants proches.

Dans le trade off entre le coût de transport, le coût foncier et la valeur des aménités et des nuisances, qui explique le choix d'une localisation résidentielle, le rôle des variables environnementales, paysagères en particulier, est difficile à évaluer. De manière à éclairer cette question, nous faisons un état de la littérature consacrée à l'estimation du prix des paysages dans le contexte particulier des campagnes résidentielles périurbaines. Puis, nous apportons une contribution au sujet en mettant en œuvre, résultats à l'appui, une méthode originale d'évaluation du prix des paysages ; la ceinture périurbaine de Dijon fait l'objet de cette application.

La structure de l'article s'articule selon quatre parties : après un résumé des méthodes d'analyse et d'évaluation du paysage (section 1), les résultats tirés de la littérature sur la valeur des paysages agricoles et forestiers sont présentés (section 2), puis notre application conduisant à l'estimation du prix des paysages périurbains dijonnais est expliquée (section 3). Enfin la conclusion fera l'objet de la section 4 .

\section{Le paysage et son évaluation économique}

\section{Définir le paysage}

Le terme de paysage recouvre de multiples sens, ambigus et parfois antagonistes. Étymologiquement, «le paysage, c'est ce que l'on voit du pays; ce que l'œil embrasse d'un seul coup d'oeil, le champ du regard » (Brunet et al, 1992). Bertrand (1978) et Berque (1991) précisent que le plus simple et le plus banal 
des paysages est à la fois social et naturel, subjectif et objectif, production matérielle et culturelle, réel et symbolique. Le paysage prend corps dans l'interaction entre un objet, l'espace/le lieu, et un sujet, l'observateur. Précisions que, si les autres sens (l'ouie, l'odorat ou même le toucher) peuvent être mobilisés dans la perception du paysage, la vue reste tout de même déterminante et restera seule en cause dans nos investigations. Le paysage apparaît comme une représentation de la réalité telle qu'elle est perçue par les sens puis interprétée par le sujet, avec son cœur et sa raison. Il fournit des informations sur un espace circonscrit dans ses trois dimensions, celui qu'un observateur perçoit du sol, dans une vision nécessairement contrainte par des volumes, des plans verticaux, des écrans, des perspectives, des angles de vision (Pinchemel, 1992).

Un tour d'horizon sans visée exhaustive permet de dégager quelques types d'approches caractéristiques du paysage.

- L'écologue interprète le paysage comme le signe de systèmes en interaction, révélateur de leurs modalités de fonctionnement faites d'échanges d'énergie, de matière ou d'information (Burel et Baudry, 1999). - L'historien ou l'ethnologue sont guidés, dans leur lecture du paysage, par la recherche des traces que les sociétés passées y ont laissées ; à ce titre, le paysage constitue un héritage et donc un point d'appui pour comprendre les interactions de tout ordre (économiques, culturelles, mythiques) que les sociétés anciennes ont entretenues avec leur cadre de vie (Ythier, 1995).

- Pour le géographe, le paysage est inscrit dans la tradition de sa démarche dont il constitue l'objet par excellence puisqu'il est l'expression résultante sensible du jeu combiné des systèmes spatiaux naturels et/ou anthropiques (Rougerie et Beroutchachvili, 1991).

- Les psychologues et les spécialistes de la cognition s'intéressent à la manière dont le paysage, objet perçu, conduit à des représentations où la dimension mentale prend le pas sur la réalité matérielle (Roger, 2001).

L'économiste s'intéresse au paysage parce qu'il induit des comportements qui lui confèrent un prix. Il s'agit d'un bien non marchand qui est un bien public plus ou moins pur. Nous nous intéressons ici à la valeur des paysages qui s'offrent à la vue des habitants depuis leur domicile. Dans ce cas, ce bien n'est pas libre d'accès, puisqu'il faut acquérir une parcelle pour en bénéficier, ce qui le rapproche d'un bien privé. Mais il n'est pas détruit dans l'acte de consommation (le paysage ne s'abîme par du seul fait qu'on le regarde) et il n'est pas toujours possible d'en exclure d'autres consommateurs, ce qui le rattache aux biens publics. Il relève des méthodes d'évaluation des biens non marchands, qu'il s'agisse de celles des préférences déclarées au cours d'enquêtes ou de celles des préférences révélées par le marché. Dans les deux cas, les résultats font ressortir le prix consenti pour jouir des paysages, les sauvegarder ou les embellir. Dans ce registre, notre ambition est d'établir une méthode qui permette de mettre en évidence, sur des bases reproductibles, le prix qui est accordé sur le marché du logement aux caractéristiques visuelles du paysage.

\section{Objectiver le paysage}

Pour procéder à l'évaluation économique des paysages, il importe que la qualité de l'information paysagère soit garantie par un protocole rigoureux. Pour cela, la formalisation géographique offre des outils géomatiques permettant d'exprimer la réalité visuelle du paysage sous la forme d'indices quantitatifs. Les principes suivants fondent cette approche :

(i) le paysage est partout; il s'inscrit dans la continuité de l'espace et du temps ;

(ii) le paysage est composé d'une infinité de points de vue qui méritent tous d'être pris en compte a priori, sans critères sélectifs d'ordre culturel ou esthétisant ;

(iii) le paysage peut être décrit par une nomenclature hiérarchisée où sont distingués objets et éléments d'image. 
Sur cette base, deux catégories d'information majeures permettent d'approcher la réalité visuelle des paysages : d'une part, le relief qui fixe son armature et l'installe dans les trois dimensions du volume scénique et, d'autre part, l'occupation du sol qui l'habille en se superposant aux formes topographiques.

Combinée au relief et déclinée dans ses différents thèmes (bâti, forêts, cultures, etc.), l'occupation du sol confère sa diversité physionomique au paysage en terme de formes, de couleurs et de textures. Il en résulte des agencements visuels plus ou moins complexes, tantôt uniformément déroulés jusqu'à l'horizon, tantôt rompus par des lignes de plan successives; les vues peuvent être dominantes et ouvrir sur de larges panoramas ou encore dominées par de hautes formes encadrantes.

Ainsi composé, le paysage s'offre au regard en tant qu'image, laquelle, une fois perçue dans sa richesse sensorielle, est ensuite interprétée et mentalement reconstruite par un sujet dans un processus de représentation. Le paysage donne donc lieu à des lectures variables d'un individu à l'autre, voire d'un moment à un autre, mais les appréciations individuelles se rejoignent en fonction d'archétypes ou de références communes liées à la culture, la formation, l'appartenance sociale, etc. Des régularités de comportement apparaissent ainsi, conduisant à valoriser ou dévaloriser certaines formes paysagères dûment caractérisées.

\section{L'évaluation économique du paysage}

Pour évaluer des biens non marchands, comme un paysage, les économistes disposent de méthodes des préférences révélées, qui déduisent le prix de ce bien du prix de la dépense consacrée à son acquisition ou à l'achat d'un bien qui le contient (prix hédonistes, coûts de transport) et de méthodes des préférences déclarées, procédant par enquête, parmi lesquelles la méthode d'évaluation contingente est la plus répandue.

Ce ne sont pas les mêmes valeurs qui sont estimées dans chacun des cas. Les méthodes de préférences révélées fournissent des valeurs d'usage (résidentielle avec les prix hédonistes, récréative avec les coûts de transport) alors que les préférences déclarées peuvent donner des valeurs de nonusage (valeurs d'existence ou de legs), mais sans décomposition selon des attributs. Nous allons, par la suite, nous centrer sur la méthode des prix hédonistes, qui est adaptée à notre objet. C'est pourquoi nous ne nous arrêterons pas aux problèmes de méthode propres à l'évaluation contingente ${ }^{1}$ ni aux résultats qu'elle permet d'obtenir sur le consentement à payer pour les paysages ${ }^{2}$.

Deux difficultés se présentent à l'économiste qui souhaite évaluer le paysage à travers un prix, exprimé en euros. La première n'est qu'apparente. Elle tient au caractère individuel de l'appréciation esthétique : la beauté n'est pas réductible à un prix, à l'instar de la douleur, de la vie, etc. Cette objection peut toutefois être levée : une toile de maître, par exemple, est évaluée, y compris celles qui ne passeront jamais sur le marché (par exemple à travers le montant de la prime d'assurance pour leur transport). Les salles de vente sont un marché où joue une concurrence quasi parfaite où se détermine le prix des œuvres d'art, par-delà les appréciations subjectives et esthétiques propres à chacun des enchérisseurs. La différence avec un paysage est que celui-ci ne passe pas sur un «marché des paysages ». Mais, comme nous allons le voir, il existe un marché indirect qui permet d'en déterminer le prix.

La seconde difficulté est plus sérieuse. L'évaluation du prix d'un paysage particulier est généralement d'un faible intérêt, sauf pour celui qui en jouit. Il faut, pour répondre à des questions d'ordre plus général, considérer, non plus un paysage singulier, mais une collection de paysages assortie de critères

1. Sur le sujet, voir Amigues et al. (1996)

2. Sur cette question, Rambonilaza (2004) présente une abondante bibliographie. 
permettant de les classer et de les comparer sur des bases assurées et quantifiées. En France, Brossard et Wieber $(1980$; 1984) ont jeté les bases d'une approche qui répond à cette exigence en se référant aux apports de la systémique. Avec les développements qui ont suivi, des procédures d'inventaire paysager ont été établies grâce aux ressources de la modélisation spatiale et de la géomatique (Brossard et al, 1995 ; Brossard et Joly, 1999 ; Foltête, 1999).

\section{Le prix hédoniste de paysages et espaces verts}

\section{La couverture des sols}

Les évaluations économiques du prix de l'occupation du sol par différents types de couverts sont nombreuses. Par exemple, des Rosiers et al. (2002) montrent que, dans la région de Québec, des arbres ont un impact positif sur le prix des maisons $(0,2 \%$ de plus pour $1 \%$ d'arbres en plus). Les aménités florales et jardinières et les haies ont aussi un effet positif. Au total, les attributs paysagers représentent en moyenne $7,7 \%$ du prix des logements. Kestens et al. (2004), dans la même région, montrent les effets positifs d'un indice synthétique de présence végétale dans un cercle de 40 mètres. Dans ces travaux, les résultats significatifs correspondent à des cercles étroits autour des maisons : de l'ordre de quelques dizaines de mètres.

Plutôt que d'énumérer un grand nombre de références de ce type, qui concluent souvent (mais pas toujours) que les espaces verts ont des prix hédonistes positifs, nous préférons insister ici sur quelques aspects particuliers. Au-delà de la couverture des sols, en particulier végétale, le statut juridique des terrains influence les prix immobiliers : dans le Maryland, la probabilité qu'un terrain soit ultérieurement construit, saisie par son statut juridique actuel, influence négativement le prix (Irwin, 2002). Cheshire et Sheppard (1995) obtiennent des résultats voisins dans les régions de Reading et Darlington (Royaume-Uni), le statut juridique indiquant ici la possibilité de pénétrer (statut public) ou non (terres agricoles privées) dans ces espaces verts.

De nouvelles méthodes de déclaration des préférences se développent, comme l'analyse conjointe utilisée par Roe et al. (2004) qui, dans l'Ohio (États-Unis), montrent un goût pour la présence de cultures et de prairies dans un rayon d'un mile autour des maisons : pour $10 \%$ de plus d'agriculture ou de cultures permanentes, le prix d'une maison augmente de $5 \%$ (3\% pour les pauvres, $6 \%$ pour les riches). Ces auteurs montrent que les aménités agricoles jouent un rôle attractif dans la suburbanisation et qu'il y a substituabilité entre terres agricoles (qui ont une valeur esthétique et de non-usage) et parcs publics (qui ont, en plus, une valeur récréative). En France, Dachary-Bernard (2005) utilise la même méthode pour le parc régional naturel d'Armorique. Les visiteurs sont prêts à payer pour que les bâtiments agricoles soient intégrés aux paysages et pour que la lande soit boisée, résultat paradoxal car les gestionnaires du parc la maintiennent dans son état patrimonial de lande rase.

\section{La composition paysagère et l'écologie des paysages}

Pour aller au-delà de l'occupation des sols et se rapprocher de la complexité de formes qu'offre un paysage, les économistes utilisent souvent des photographies. C'est ainsi que Palmer (2003) demande à des enquêtés de noter des photos du Cap Cod (Dennis, Massachusetts, États-Unis), pour conclure qu'ils valorisent l'eau, mais qu'ils dévalorisent les forêts et sont indifférents à leur aspect esthétique. Ces auteurs calculent des indices d'écologie du paysage (taille de la tache la plus grande, nombre de taches par hectare, longueur des frontières par hectare, richesse, indice de Shannon), qui n'ont pas d'impact significatif sur la valeur, à l'exception de la longueur des haies, faiblement valorisée. 
Cette méthode photographique est très lourde dès que la zone d'étude est un peu étendue. C'est pourquoi les chercheurs se sont tournés vers les images satellites dès qu'elles ont été accessibles. L'écologie du paysage a été la discipline la plus active en ce domaine : la première publication résulte d'un programme interdisciplinaire d'écologie du paysage et d'économie (Geoghegan et al, 1997). Elles utilisent des indices d'écologie du paysage et une fonction de prix hédoniste pour un bassin hydrographique du Maryland proche de Washington D.-C. (États-Unis). Acharya et Bennett (2001) réalisent un travail très proche dans un bassin hydrographique du Connecticut (États-Unis). Les variables sont calculées dans des cercles de quelques centaines de mètres autour du logement (en supposant que cela correspond au champ de vision) et d'un à deux kilomètres (le champ d'une promenade pédestre). Autour de Washington, la proximité immédiate d'espaces agroforestiers accroît (faiblement) les valeurs foncières, mais l'effet devient négatif lorsqu'ils sont plus distants. La fragmentation et la diversité du paysage autour du logement ont un effet négatif sur les valeurs foncières, sauf très près et très loin de Washington.

Pour ce qui concerne la France, Dumas et al. (2005) ont appliqué un modèle de prix hédoniste au département des Bouches-duRhône (en excluant les villes) en introduisant des variables d'occupation des sols et paysagères, ces dernières obtenues à dire d'experts ou calculées par des indices d'écologie du paysage. Sans prendre en compte ces derniers, c'est le tissu urbain continu qui est le mode d'occupation des sols dont le prix hédoniste est le plus élevé, suivi du bâti isolé et de terrains mixtes agricoles et résidentiels, alors que les forêts ont un signe négatif, comme les zones industrielles et les décharges. Cependant, l'ajout dans la régression des indices paysagers change le signe du tissu urbain continu, des zones agricoles et des espaces ouverts, qui devien- nent tous négatifs. L'indice d'agrégation a un prix hédoniste positif, ceux d'interspection, de juxtaposition et de Shannon des prix négatifs. Les résultats sont donc assez dépendants de la spécification, probablement du fait de liaisons entre les variables d'occupation des sols et les indices d'écologie des paysages.

\section{La distance à des couverts agroforestiers}

La distance entre un logement et un objet « vert » est assez aisée à calculer sur un plan ou une carte. C'est pourquoi les économistes ont intégré depuis longtemps ce type de variable. C'est ainsi que Bolitzer et Netusil (2000), dans la région de Portland (Oregon, États-Unis), montrent que les logements situés à moins de 500 mètres à vol d'oiseau d'une zone verte valent $1,5 \%$ plus cher que la moyenne et qu'une trop grande proximité a des effets négatifs (congestion, bruits). Smith et al. (2002) montrent qu'en Caroline du Nord (États-Unis) l'éloignement du golf le plus proche a un effet négatif sur le prix des maisons et que le fait d'être sur une parcelle qui lui est adjacente est positivement valorisé. De plus, les ménages préfèrent ne pas être adjacents à une parcelle agricole.

Thorsnes (2002) analyse l'impact de la forêt sur les valeurs immobilières dans la région des Grands Rapides du Michigan (États-Unis). Les biens qui jouxtent une forêt valent de 19 à $35 \%$ de plus que les autres. En revanche, de l'autre côté de la rue, la forêt n'a plus d'effet sur le prix. Sur cette même question de la contiguïté, Hobden et al. (2004) montrent, pour une ville moyenne à 25 kilomètres de Vancouver (Canada), que les parcelles résidentielles mitoyennes d'espaces verts valent $2,8 \%$ de plus que les autres. Les petits espaces verts, qui sont généralement des corridors conduisant à de grands parcs, augmentent la valeur de 6,9 \% et lorsqu'il y a en plus un sentier piétonnier la plusvalue atteint $11 \%$. 
Tyrvainen et Miettinen (2000), concluent que, dans une petite région peu peuplée au nord d'Helsinki (Finlande), le prix des maisons diminue de 5,9\% par kilomètre d'éloignement du parc forestier le plus proche et que la vue sur des forêts a un prix hédoniste correspondant à 4,9\% du prix du logement. L'effet de la proximité est significatif au seuil de $1 \%$ à moins de 600 mètres, puis entre 600 mètres et un kilomètre, il n'est significatif qu'au seuil de $5 \%$ et il ne l'est plus au-delà.

\section{La vue du paysage}

\section{L'analyse des préférences en matière} paysagère

Les travaux qui étudient les préférences des consommateurs sans réaliser d'évaluation économique du prix des attributs expliquent, le plus souvent, une note donnée par un jury à une photo de paysage par des caractéristiques de celui-ci. Beaucoup d'entre eux sont anciens, mais on trouve aussi des études récentes. Les références sont très nombreuses et les conclusions ressemblent à un fouillis inextricable. C'est ainsi que Gobster et Chenoweth (1989), qui citent plus de 80 références et relèvent 1194 termes utilisés dans cinquante papiers pour décrire les préférences esthétiques (même après regroupement, il reste 114 attributs différents).

Kaplan et al. (1989), souvent cités dans la littérature, comparent le rôle de quatre ensembles de variables dans l'explication d'une note de photos de paysages : attributs physiques (pente, relief, contraste, diversité), occupation des sols, variables informationnelles (se rapportant aux perceptions et sensations) et, enfin, de perception (champ visuel, texture). Dans la zone rurale du Michigan (États-Unis) étudiée, les attributs physiques sont peu explicatifs. Les pelouses arborées ont un effet positif sur la note, l'agriculture, comme les broussailles ou les friches, un effet négatif et les autres types (forêts, prairies) sont neutres. Le mystère a un bon effet prédictif, ce qui est un résultat classique (selon les auteurs), de même que l'ouverture (dont l'effet est négatif, ce qui les surprend).

Des études de ce type sont également réalisées en Europe. Par exemple, Arriaza et al. (2004) montrent que, dans la région de Cordoue (Espagne), l'état sauvage, l'eau et les arbres sont des attributs valorisés, ainsi que le contraste des couleurs. De Groot et van den Born (2003) étudient les goûts des Hollandais d'une petite région rurale (Gennep) et montrent que la majorité des enquêtés préfèrent une nature plus ou moins sauvage à une nature apprivoisée ou arcadienne (parc-like).

\section{L'estimation du prix de paysages vus}

Le travail réalisé par Bastian et al. (2002) sur la valeur de la vue dans les Montagnes Rocheuses (Wyoming) est le premier, à notre connaissance, qui combine une méthode de géographie quantitative, qui reconstitue la vue d'un paysage, et une méthode économétrique de prix hédoniste. L'environnement de 158 parcelles est caractérisé à partir d'images satellites permettant de définir la couverture des sols et d'un modèle numérique de terrain qui donne la topographie et les masques visuels du relief. L'aire de vision sur $360^{\circ}$ est analysée autour de chaque point, dans une vision planimétrique (map-like) et panoramique (photograph-like). Les résultats montrent que les biotopes d'habitat des élans et les rivières à truites ont des prix hédonistes positifs et que la diversité des paysages est appréciée, mais que des variables de vue (surface vue, relief, longueur des haies, etc.) ne sont pas significatives.

Paterson et Boyle (2002) combinent la couverture des sols et le paysage vu, en examinant les relations entre ces deux variables dans une région rurale du Connecticut (États-Unis) à partir d'images satellites et d'un modèle numérique de terrain qui permet de déterminer le champ visuel. Les résultats diffèrent selon le type de vision (depuis le satellite ou depuis le sol). 


\section{Le prix des paysages périurbains dijonnais}

Dans cette recherche ${ }^{3}$, nous empruntons aux différents courants de la géographie précédemment évoqués : un paysage est la combinaison d'un système émetteur (ses composantes physiques) et d'une personne, qui le perçoit à travers des filtres qui lui sont propres. On peut dire, en pensant à Lancaster (1966), que la vue d'un paysage est l'output d'une opération de production individuelle qui utilise un système émetteur (input, i.e. les composantes physiques) pour produire de l'utilité grâce à des outils sensoriels et intellectuels. Nous évaluons le prix de ces attributs par le programme microéconomique classique de maximisation de l'utilité sous contrainte budgétaire ce qui se traduit par la mise en œuvre de la première étape de la démarche à la Rosen (1974).

\section{Les modèles économétrique et géographique}

L'encadré 1 décrit les aspects essentiels du modèle économétrique. Il s'agit d'un modèle à effet fixe estimé en différences (pour tenir compte des autocorrélations spatiales) par la méthode des variables instrumentales (pour tenir compte de l'endogénéité de variables explicatives). L'encadré 2 explique la modélisation du paysage.

\footnotetext{
3. $C f$. Cavailhès et Joly (2006) pour une présentation détaillée de cette recherche qui, d'une part, a bénéficié du financement de la Région de Bourgogne, du Conseil général de la Côte d'Or et de la Communauté d'agglomération de Dijon et qui, d'autre part, utilise des données sur les transactions immobilières en provenance des notaires (société Perval).
}

\section{Encadré 1. Le modèle économétrique}

Il existe des liaisons spatiales entre les résidus, qui sont dues à des caractéristiques omises que partagent des observations voisines. Pour en tenir compte, nous définissons un groupe, qui est un lotissement ou le lotissement fictif constitué par les observations d'une commune située hors lotissement. Les observations d'un même groupe partagent une politique foncière locale (imposition, zonage foncier), une qualité sociale du voisinage, une jouissance de biens publics locaux, une accessibilité aux marchés du travail, des biens et des services, la présence d'aménités, nuisances et externalités diverses. De plus, deux lotissements voisins (ou deux communes voisines) ont souvent des caractéristiques différentes en ces domaines. Pour tenir compte de ces liaisons spatiales, nous choisissons un modèle où la variable endogène est la différence entre le logarithme du prix d'une maison et le logarithme du prix moyen des maisons du lotissement auquel elle appartient. Elle est expliquée par la déviation des variables explicatives par rapport à la moyenne du lotissement. Il s'agit donc d'un modèle à effet fixe estimé en différences.

Un test de Hausman montre que la surface habitable des logements est endogène, ce que nous corrigeons en utilisant la méthode instrumentale : estimation en doubles moindres carrés après projection de la surface habitable sur des instruments (caractéristiques des acquéreurs et vendeurs). Le test de Sargan indique qu'il n'y a pas d'autre variable endogène. Le terme d'erreur est hétéroscédastique, mais l'estimateur des moindres carrés généralisés est le même que celui des moindres carrés ordinaires (Sevestre, 2002). L'incomplétude du panel (i.e. un nombre d'observations variable d'un lotissement à l'autre) est une autre source d'hétéroscédasticité qu'il est facile de corriger (Sevestre, op. cit.). Le test de White indique que les résidus sont homoscédastiques. 


\section{Encadré 2 . La modélisation du paysage}

Principes. Notre méthode d'analyse du paysage, fondée sur un système d'information géographique (SIG) qui gère des données multi-sources, parmi lesquelles les images de satellites et un modèle numérique de terrain (MNT), est la suivante :

(i) Nous formalisons les relations d'intervisibilité entre un observateur et le paysage donné à voir. Nous montrons ainsi la différence qui existe entre la « vue du dessus », comme celle d'une photographie aérienne, et la « vue du dedans », perçue depuis le sol, d'où elle peut buter sur le relief et sur des objets hauts qui ferment l'horizon.

(ii) Un paysage est organisé en plans de vision, du proche au lointain. Un objet, comme un arbre, n'a pas le même sens pour l'observateur selon qu'il est près (il est alors un objet singulier) ou loin (il se fond le plus souvent dans une forêt). Nous tenons compte de cette propriété en utilisant des méthodes multi-échelles qui font varier la nature des objets selon la distance.

(iii) La vue d'un paysage peut être une aménité, mais la possibilité d'être vu par autrui (le passant sur une route, l'habitant d'une maison voisine) peut être une nuisance. Or la première, ou vue active, n'est pas symétrique de la seconde, ou vue passive, du fait de masques (une forêt peut être vue de loin, mais le promeneur qui s'y trouve ne voit qu'un faible espace de sous-bois). Nous introduisons cette distinction entre « vue active » et « vue passive».

Données. Nous utilisons les ressources de deux capteurs donnant des images à haute résolution (le système Enhanced Thematic Mapper du satellite Landsat 7 et le système Indian Remote Sensing) et la base européenne Corine Land Cover, de plus faible résolution. La base vectorielle diffusée par l'Institut géographique national (IGN), appelée BD CARTO ${ }^{\circledR}$, permet d'identifier des éléments linéaires comme les routes, les voies ferrées et les rivières ou des éléments ponctuels comme les carrières ou les zones d'activités, difficiles à identifier par télédétection. Le modèle numérique de terrain (MNT) est tiré d'une base appelée BD ALTI ${ }^{\oplus}$ produite par l'IGN à la résolution de 50 mètres. Un modèle numérique d'élévation (MNE) est constitué en ajoutant, aux altitudes du MNT, une hauteur standard pour chaque type d'objet $(20 \mathrm{~m}$ pour les résineux, $15 \mathrm{~m}$ pour les feuillus, $3 \mathrm{~m}$ pour les buissons, $1 \mathrm{~m}$ pour les vignes, $7 \mathrm{~m}$ pour le bâti, 0 pour les autres types). Ces données permettent de reconstituer le paysage vu à un moment donné (en 2000), mais pas sa dynamique (alors que les ménages anticipent peut-être une évolution au moment de l'achat). Les données sont structurées selon quatre niveaux de résolution, soit 7, 30, 150 et 1000 mètres.

Mise en œuvre. La conception et la mise en œuvre de noyaux logiciels spécifiques ont été nécessaires pour paramétrer et simuler les relations d'intervisibilité et pour réduire le temps de calcul $^{(+)}$. On mesure ainsi la surface vue par un observateur virtuellement placé en chaque pixel et qui porte son regard sur les 360 degrés du tour d'horizon (vu actif). L'opération consiste à dénombrer les pixels vus, du proche au lointain, en tenant compte du relief et de la hauteur des objets. Pour cela, des rayons divergents, espacés de 3 degrés, sont lancés à partir du pixel de base. On détermine, par un calcul trigonométrique, si les pixels rencontrés au long de ces rayons sont vus ou non, en tenant compte de l'altitude et de la hauteur des objets. La même opération est répétée pour chaque pixel. Une démarche symétrique est réalisée pour le vu passif. Un paysage virtuel est ainsi obtenu pour chaque pixel de l'aire d'étude. Le géoréférencement des points où sont situées les maisons permet de connaître le paysage en chacun de ces points.

(*) Cf. (Joly et al., 2006, "Using modeling to make a quantitative valuation of landscape", Besançon, Dijon, CNRS-ThéMA, INRA-CESAER, document de travail, p. 9) : «A difficulty arising from landscape modeling such as we have presented it, is due to the fact that the limit of the visual field cannot be fixed a priori. The ideal would be to carry out an exhaustive visibility test on all the points of a matrix in order to take into account the whole of the visual space. This procedure is not used for practical purposes since it would require the iteration of calculations several millions of times on several million pixels (an image at a resolution of 7 meters is composed of 130 million pixels) ». 
L'encadré 2 précise la méthode géographique. Depuis chaque point ${ }^{4}$ de la zone d'étude, nous étudions le tour d'horizon sur $360^{\circ}$ en lançant des rayons et en testant le long de ces rayons le type d'objet qui occupe chaque pixel rencontré (qui sont ici des carrés de 7 mètres de côté). En tenant compte du relief et de la hauteur des objets ainsi repérés, le nombre de pixels vus le long du rayon est calculé. Par sommation, on connaît le champ de vision et la nature des pixels qui s'y trouvent, qui sont les éléments de paysage utilisés par le modèle économique. Douze types d'occupation du sol sont ainsi identifiés : eau, résineux, feuillus, buissons, labours, prés, vignes, routes, bâti, carrières, voies ferrées et zones d'activité.

Grâce à ces données, nous modélisons dans des couronnes concentriques autour de chaque pixel :

(i) l'occupation du sol vu depuis le satellite (vue d'en haut),

(ii) le paysage tel qu'il est vu depuis le sol, (iii) la soumission à la vue d'autrui.

La distinction de ces trois aspects n'a pas encore, à notre connaissance, été introduite dans l'évaluation économique des paysages, alors que nos résultats montrent qu'elle est importante.

La région d'étude est délimitée par un temps d'accès à Dijon inférieur à 33 minutes ou une distance routière de moins de 42 kilomètres. Elle englobe 14 communes de la banlieue de Dijon (la commune de Dijon est exclue de l'étude), que nous appellerons le «pôle urbain » dans ce qui suit et qui compte 87086 habitants, et 305 communes périurbaines ou rurales (nous parlons de la « ceinture périurbaine », qui compte 140703 habitants). Il s'agit d'une zone en croissance démographique, en particulier la ceinture périurbaine. De ce fait, les paysages analysés à partir du passage des satellites en

4. La longitude et la latitude de chaque point, y compris ceux où sont localisées les transactions immobilières, sont géoréférencées dans un système de coordonnées cartésiennes (Lambert).
2000 peuvent évoluer, sans qu'il soit possible de tenir compte de cet aspect dynamique.

Les données et les variables économiques proviennent des notaires (S.A. Perval). La base de données est constituée de 4734 ventes de maisons individuelles intervenues entre 1995 et 2002 . Il s'agit de ventes entre particuliers pour lesquelles les notaires relèvent le prix de la transaction et certaines caractéristiques du bien et des personnes. Les estimations ont été faites sur 4050 observations $^{5}$.

\section{Résultats}

Le modèle donne un assez bon ajustement d'ensemble (le $\mathrm{R}^{2}$ est de 0,53 ). Les prix hédonistes des variables de structure et des caractéristiques de la transaction (non repris ici) sont cohérents avec ceux obtenus par ailleurs. Le tableau 1 indique les résultats concernant les variables paysagères qui ont des prix hédonistes significatifs, pour l'ensemble des observations et en distinguant celles du pôle urbain (les 14 communes de la banlieue dijonnaise) et de la ceinture périurbaine.

Retenons, tout d'abord, que les ménages sont « myopes », c'est-à-dire que les plans de vision situés au-delà de quelques centaines, voire de quelques dizaines de mètres et les objets qu'ils contiennent ont des prix hédonistes non significatifs : la plupart des résultats significatifs concernent les 70 premiers mètres autour des maisons. Notons également que la plupart des variables retenues dans les régressions n'influencent pas significativement le prix des maisons dans le pôle urbain, contrairement à la ceinture périurbaine. Cela peut s'expliquer par les limites de notre méthode (peu adaptée aux paysages urbains) ou par

5. Sur l'ensemble de ces observations, 684 ont été éliminées pour deux types de raisons : observations atypiques ou insuffisances de la base de données (variables non renseignées ou erreurs de saisie) ou lotissements avec une seule observation. 
un tri du marché conduisant les ménages sensibles aux aménités paysagères « vertes » vers des localisations périurbaines. Quoi qu'il en soit, nous nous attacherons par la suite à commenter les résultats de cette ceinture périurbaine.

Un are de feuillus supplémentaire vu dans les 70 premiers mètres autour d'une maison a un prix hédoniste qui dépasse $1100 €$ dans la ceinture périurbaine. Les maisons avec vue sur des feuillus en voient en moyenne 3,5 ares dans les 70 premiers mètres, ce qui signifie qu'un doublement de cette quantité a un prix hédoniste de $3550 €(3,4 \%$ du prix d'une maison). Les résineux, moins abondants dans cette région, donnent des résultats voisins. La forme des feuillus a un effet significatif sur le prix qui s'ajoute au précédent : une tache supplémentaire de feuillus dans un rayon de 70 mètres a un prix positif de $660 €$ et, à l'inverse, le prix de la longueur des lisières de feuillus est négatif : - $25 €$ par mètre supplémentaire dans la ceinture périurbaine. La combinaison de ces deux variables donne une indication sur les formes qui sont valorisées : des taches nombreuses avec peu de lisières correspondent à de petits bosquets arrondis et non à des forêts en masse ni à des formations allongées.

Les cultures vues entre 70 et 140 mètres autour des maisons de la ceinture périurbaine ont un prix hédoniste positif de $45 €$ par are supplémentaire, soit, pour les maisons d'où l'on en voit, $1700 €$ lorsque la quantité double. Les prés ont aussi un prix positif entre 140 et 280 mètres : $16 €$ par are supplémentaire, soit $1170 €$ pour un doublement de la surface vue (lorsqu'elle n'est pas nulle). Les vignes n'ont pu être prises en compte car elles sont trop rares. Il ressort de la comparaison avec les forêts que le prix hédoniste des surfaces agricoles est positif à des distances un peu plus lointaines que celui des arbres,

Tableau 1. Prix hédoniste des attributs paysagers (€)

\begin{tabular}{|c|c|c|c|c|c|c|}
\hline & \multicolumn{2}{|c|}{ Ensemble } & \multicolumn{2}{|c|}{ Pôle urbain } & \multicolumn{2}{|c|}{ Périurbain } \\
\hline & +1 pixel & $\begin{array}{c}\text { Double- } \\
\text { ment }\end{array}$ & +1 pixel & $\begin{array}{c}\text { Double- } \\
\text { ment }\end{array}$ & + 1 pixel & $\begin{array}{c}\text { Double- } \\
\text { ment }\end{array}$ \\
\hline Feuillus (pixels, champ 0 à 70 mètres) & 451 & 2165 & NS & NS & 515 & 3553 \\
\hline Résineux (pixels, champ 0 à 70 mètres) & 1196 & 2991 & NS & NS & 1755 & 4388 \\
\hline Bâti : écart soumission - vue (pixels, champ 0 à 70 mètres) & -915 & -823 & NS & NS & -1212 & 1333 \\
\hline Taux bâti (champ 0 à 70 mètres) & 401 & 522 & 2196 & 3513 & NS & NS \\
\hline Routes (pixels, soumission depuis champ 0 à 70 mètres) & -48 & -874 & \multicolumn{2}{|c|}{ idem ensemble } & \multicolumn{2}{|c|}{ idem ensemble } \\
\hline Prés (pixels, champ 70 à 280 mètres) & 8 & 147 & NS & NS & 8 & 1170 \\
\hline Cultures (pixels, champ 70 à 140 mètres) & 13 & 271 & NS & NS & 22 & 1702 \\
\hline Voies ferrées (pixels, champ 0 à 280 mètres) & -258 & -2054 & \multicolumn{2}{|c|}{ idem ensemble } & \multicolumn{2}{|c|}{ idem ensemble } \\
\hline Eaux (pixels, champ 70-280 mètres) & -90 & -2094 & \multicolumn{2}{|c|}{ idem ensemble } & \multicolumn{2}{|c|}{ idem ensemble } \\
\hline Moins 200 mètres route grande circulation & -4976 & & NS & & -8140 & \\
\hline Pente (pour cent) & -1201 & -2883 & \multicolumn{2}{|c|}{ idem ensemble } & \multicolumn{2}{|c|}{ idem ensemble } \\
\hline Déviation par rapport à l'orientation Sud (degré) & -16 & -441 & NS & NS & -36 & -1005 \\
\hline Indice division (1 pour cent) & 109 & & NS & & 322 & \\
\hline Indice contiguité (1 pour cent) & -117 & & NS & & -144 & \\
\hline Nombre taches feuillus & 656 & & \multicolumn{2}{|c|}{ idem ensemble } & \multicolumn{2}{|c|}{ idem ensemble } \\
\hline Tache dominante & 86 & & NS & & 209 & \\
\hline Longueur lisière feuillus (mètres) & -12 & & 26 & & -25 & \\
\hline
\end{tabular}

NS : non significatif au seuil de $5 \%$.

Lecture : le prix hédoniste d'un pixel supplémentaire de feuillus (soit $49 \mathrm{~m}^{2}$ ) est de 451 € (non significatif dans le pôle urbain, $515 €$ dans le périurbain) ; pour un doublement du nombre de pixels, le prix est de $2165 €$ (non significatif dans le pôle, $3553 €$ dans le périurbain). 
quoiqu'elles restent circonscrites dans un rayon de deux ou trois centaines de mètres.

La distinction entre la vue de bâti et la soumission à la vue depuis des pixels bâtis est importante : la première est une aménité et la seconde une nuisance. C'est ce que montre, dans le tableau 1, la différence entre le nombre de pixels bâtis d'où l'on peut être vu et le nombre de pixels bâtis qui sont vus : pour une différence d'un are, le prix d'une maison baisse de $2400 €$ dans la ceinture périurbaine. Les réseaux de transport ont des effets significatifs sur le prix, soit du fait de leur vue $(-100 € /$ are pour les routes, $-520 € /$ are pour les voies ferrées) soit par leur simple proximité (ruban de moins de 200 mètres autour des routes à grande circulation). La pente de la parcelle résidentielle a un prix négatif, qui est légèrement plus bas lorsqu'elle n'est pas orientée au sud.

Les pixels non vus à proximité des maisons (masqués par le relief ou des objets hauts) ont des effets non significatifs. C'est donc la vue proprement dite qui compte : des pixels arborés et des routes ont des prix hédonistes respectivement positif et négatif lorsqu'ils sont vus, mais ces prix ne sont pas significatifs lorsque ces objets existent à proximité des maisons sans être vus. Dans le même ordre d'idée, pour corroborer l'importance de la vue elle-même, les indices d'écologie du paysage indiquent une valorisation positive des formes paysagères complexes (nous avons retenu des indices de division et de non-contiguïté dans le tableau 1).

En utilisant la méthode des indices de quality of life (Blomquist et al, 1988), les prix hédonistes des attributs paysagers permettent de calculer un prix global des paysages, égal à la somme des quantités pondérées par les prix. Pour la ceinture périurbaine, ce prix moyen est de $2400 €(2,2 \%$ du prix d'une maison) et le prix médian de $1000 €(1,2 \%)$. Ce prix augmente de 35,80 € lorsqu' on s'éloigne d'un kilomètre de Dijon, ce qui permet de penser que les paysages contribuent à expliquer la périurbanisation. En effet, lorsque les aménités paysagères augmentent avec la distance, la courbe de rente foncière est moins pentue et le peuplement est plus étendu que dans le cas d'un espace homogène.

\section{Conclusions}

De nos jours, la vue d'un paysage est encore difficile à traduire en variables économiques. Il s'agit d'un domaine qui évolue rapidement et dont les résultats ne sont pas encore fixés ni stabilisés. Il semble cependant que la vue proprement dite doit être prise en compte, car la vision qu'à un observateur depuis le sol est très différente de la vue depuis le ciel. Or, si la méthode photographique est utilisée depuis longtemps pour donner à voir un paysage à un enquêté, la télédétection, de plus en plus utilisée, offre le plus souvent une vision très éloignée de la perception courante, sauf dans de rares études (parmi lesquelles l'étude de la région dijonnaise que nous avons présentée).

Retenons, en termes de contenu, premièrement, que le prix du paysage n'est qu'une faible composante du prix du logement : de l'ordre de quelques pour cent (pour prendre une fourchette large, entre 1 et $5 \%$ ). Deuxièmement, la localisation de ces espaces verts et ouverts dans le système urbain joue un rôle dans la détermination de leur prix, qui décroît avec la distance. Troisièmement, les effets paysagers sur le prix des logements sont en général très localisés : quelques dizaines de mètres, une ou deux centaines tout au plus. Quatrièmement, la proximité de la forêt est fortement appréciée alors qu'il semble que, pour l'agriculture, une certaine distance soit préférable : ce sont plutôt les champs et les prés vus à quelques centaines de mètres qui apportent une plus-value immobilière.

Finalement, plusieurs des travaux concluent que la recherche d'aménités paysagères contribue à expliquer la périurbanisation. Les causes de l'extension des 
villes vers leur hinterland ont longtemps été recherchées du côté du push, la dégradation des centres urbains, la ghettoïsation et la criminalité des métropoles américaines repoussant la middle class vers les suburbs (Mieszkowski et Mills, 1993). Aujourd'hui, c'est le pull qui retient l'attraction.

Aux États-Unis, selon le Trust for Public Land, sur 1376 referendums locaux consacrés aux plans urbains dans 43 États (entre 1996 et 2002), 1065 ont approuvé des mesures de sauvegarde d'espaces ouverts, pour un total de 27 millions de dollars. Lors des élections du 4 novembre 2004, 120 votes sont intervenus dans 26 États pour affecter 3,25 millions de dollars à la protection d'espaces ouverts. En 1998, le NewJersey a fait l'acquisition du cinquième de la superficie de cet État pour le prévenir d'une urbanisation future. Cet engouement pour le vert, en franchissant l'Atlantique, est maintenant arrivé en Europe.

\section{RÉFÉRENCES BIBLIOGRAPHIQUES}

Acharya G., Bennett L.-L. (2001). Valuing Open Space and Land-Use Patterns in Urban Watershes. Journal of Real Estate Finance and Economics, volume 22, p. 221-237.

Amigues J.-P., Desaigues B., Vuong Q.-H. (1996). L'évaluation contingente : controverses et perspectives. Cahiers d'Economie et Sociologie Rurales, Paris, Inra, $\mathrm{n}^{\circ} 39-40$.

Arriaza M., Canas-Ortega J.-F., CanasMadueno J.-A., Ruiz-Aviles P. (2004). Assessing the Visual Quality of Rural Landscape. Landscape and Urban Planning, vol. 69, p. 115-125.

Bastian C.-T., McLeod D.-M., Germino M.-J., Reiners W.-A., Blasko, B.-J. (2002). Environmental Amenities and Agricultural Land Values: a hedonic model using geographic information systems data. Ecological Economics, vol. 40, p. 337-349.

Bertrand G. (1978). Le paysage, entre la nature et la société. Revue de géographie des Pyrénées et du Sud-Ouest, $\mathrm{n}^{\circ} 49$, p. 16-26.

Berque $\mathrm{A}$. La transition paysagère comme hypothèse de projection de l'avenir de la nature. In « Maîtres et protecteurs de la nature », Paris, Éditions Champ Vallon, p. 217-237.
Blomquist G.-C., Breger M.-C., Hoehn J.- P. (1988). New Estimates of Quality of Life in Urban Areas. American Economic Review, vol. 78, p. 89-107.

Bolitzer B., Netusil N.-R. (2000). The Impact of Open Spaces on Property Values in Portland, Oregon. Journal of Environmental Management, vol. 59, p. 185-193.

Brossard T., Joly D. (1999). Représentation du paysage et échelles spatiales d'information. Revue Internationale de Géomatique, vol. 9, n 3, p. 359-375.

Brossard T., Joly D. (2000). Enjeux paysagers et systèmes d'information géographique, présentation d'un itinéraire méthodologique et technique. Communication au colloque «Paysage et Information géographique », ENGREF-ENITA, Clermont-Ferrand, 20 octobre 1998, p. 11-21; 73-76.

Brossard T., Joly D., Laffly D., Vuillod P., Wieber J.-C. (1995). Pratique des systèmes d'information géographique et analyse des paysages. Revue Internationale de Géomatique, vol. 4, n 3-4, p. 243-256.

Brossard T., Joly D., Strasfogel S., Venzac L. (1999). Évaluation et suivi des paysages par système d'information géographique, Exemple appliqué à l'arrière pays de Bourbonne-les Bains, Haute-Marne. In «Nature et Paysage », Paris, Éditions Champvallon, p. 45-62. 
Brossard T., Joly D., Wieber, J.-C. (1998). Analyse visuelle systématique des paysages de cours d'eau par deux approches complémentaires. Revue Géographique de Lyon, vol. 73, $\mathrm{n}^{\circ} 4$, p. 299-308.

Brossard T., Wieber J.-C. (1980). Essai de formulation systémique d'un mode d'approche du paysage. Bulletin de l'Association des géographes français, $\mathrm{n}^{\circ} 468$ 469, p. 103-111.

Brossard T., Wieber J.-C. (1984). Le paysage, trois définitions; un mode d'analyse et de cartographie. L'espace Géographique, p. 5-12.

Brueckner J.-K., Thisse J.-F., Zenou Y. (1999). Why is Central Paris Rich and Downtown Detroit Poor? An Amenitybased Theory. European Economic Review, 43, p. 91-107.

Brunet R., Ferras R., Théry H. (1992). Les mots de la géographie, dictionnaire critique. Paris, Reclus-La Documentation Française, 470 p.

Burel F., Baudry J. (1999). Écologie du paysage. Concepts, méthodes et applications. Éditions Tec\&Doc, 359 p.

Caruso G. (2002). La diversité des formes de la périurbanisation en Europe. In PerrierCornet Ph. (Éd), «Repenser les campagnes », DATAR, Éditions de l'Aube, p. 67-99.

Cavailhès J., Joly D. (Éds) (2006). Les paysages périurbains et leur prix. Presses universitaires de Franche-Comté, 201 p.

Cheshire P., Sheppard S. (1995). On the Price of Land and the Value of Amenities. Paris, Economica, vol. 62, p. 247-267.

Dachary-Bernard J. (2005). Une évaluation économique du paysage. Une application de la méthode des choix multi-attributs aux Monts d'Arrée. A paraître in Économie et Statistique.

De Groot W.-T., van den Born R.-J.-G. (2003). Visions of Nature and Landscape Type Preferences: an exploration in the Netherlands. Landscape and Urban Planning, vol. 63, p. 127-138.
Des Rosiers F., Thériault M., Kestens Y., Villeneuve P. (2002). Landscaping and House Values: an empirical investigation. Journal of Real Estate Research, vol. 23, p. 139-161.

Dumas E., Geniaux G., Napoleone C. (2005). Les indices de l'écologie du paysage à l'épreuve du marché foncier. Revue d'Économie Régionale et Urbaine, 1 p. 83-106.

Foltête J.-C. (1999). Description d'objets surfaciques dans une partition spatiale, application à la définition communale de l'occupation du sol en Franche-Comté. Revue Internationale de Géomatique, vol. 2, p. 153-171.

Fujita M., Thisse J.-F. (2002). Economics of Agglomeration. Cambridge, Cambridge University Press, 466 p.

Geoghegan J., Wainger L.-A., Bockstael N.-E. (1997). Spatial Landscape Indices in a Hedonic Framework: an ecological economics analysis using GIS. Ecological Economics, vol. 23, p. 251-264.

Gobster P.-H., Chenoweth R.-E. (1989). The Dimensions of Aesthetics Preference: a quantitative analysis. Journal of Environment Management, vol. 29, p. 47-72.

Hobden D.-W., Laughton G.-E., Morgan K.-E. (2004). Green Space Borders: a tangible Benefit? Evidence from Four Neighbourhoods in Surrey, British Columbia, 1980-2001. Land Use Policy, vol. 21, p. 129-138.

Irwin E.-G. (2002). The Effects of Open Space on Residential Property Values. Land Economics, vol. 78, p. 465-480.

Kaplan R., Kaplan S., Bown T. (1989). Environmental Preferences. A Comparison of Four Domains of Predictors. Environment and Behavior, vol. 21, p. 509-530.

Kestens Y., Thériault M., des Rosiers F. (2004). The impact of surrounding land use and vegetation on single-family house price. Environment and planning $B$, vol. 31, p. 539-567. 
Lancaster K.-J. (1966). A new approach to consumer theory. Journal of Political Economy, vol. 74, p. 132-157.

Le Jeannic T. (1997). Trente ans de périurbanisation : extension et dilution des villes. Économie et statistique, 307, 7, p. 21-41.

Mieszkowski P., Mills E.-S. (1993). The Causes of Metropolitan Suburbanization. Journal of Economic Perspectives, vol. 7 (3), p. 135-147.

Palmer J.-F. (2003). Using Spatial Metrics to Predict Scenic Perception in a Changing Landscape: Dennis, Massachusetts. Landscape and Urban Planning, vol. 69, p. 201-218.

Paterson R.-W., Boyle K.-J. (2002). Out of Sight, Out of Mind? Using GIS to Incorporate Visibility in Hedonic Property Value Models. Land economics, vol. 78, p. 417-425.

Pinchemel P., Pinchemel G. (1992). La face de la Terre. Paris, Armand Colin, collection U Géographie, $2^{\mathrm{e}}$ édition, $519 \mathrm{p}$.

Rambonilaza M. (2004). Évaluation de la demande de paysage : état de l'art et réflexions sur la méthode du transfert des bénéfices. Paris, Inra, Cahiers d'Économie et Sociologie Rurales, vol. 70, p. 77-101.

Roe B., Irwin E.-G., Morrow-Jones H.-A. (2004). The Effects of Farmland, Farmland Preservation, and other Neighborhood Amenities on Housing Values and Residential Growth. Land Economics, vol. 80, (1), p. 55-75.

Roger A. (2001). Le paysage n'existe pas, il faut l'inventer? In Actes du colloque international « Patrimoine et paysages culturels », Éditions Confluences, SaintEmilion, 30 mai- ${ }^{\text {er }}$ juin, Bordeaux, p. 5563.

Rosen S. (1974). Hedonic Prices and Implicit Markets: product differentiation in pure competition. Journal of Political Economy, vol. 82, p. 34-55.

Rougerie G., Beroutchachvili N. (1991). Géosystèmes et paysages : bilans et méthodes. Paris, Armand Colin, 302 p.

Sevestre P. (2002). Économétrie des données de panel. Paris, Dunod.

Smith V.-K., Poulos C., Kim H. (2002). Treating Open Space as an Urban Amenity. Resource and Energy Economics, vol. 24, p. 107-129.

Thorsnes P. (2002). The Value of a Suburban Forest Preserve: estimates from sales of vacant residential building lots. Land Economics, vol. 78, p. 626-441.

Tyrvainen L., Miettinen A. (2000). Property Prices and Urban Forest Amenities. Journal of Environmental Economics and Management, vol. 39, p. 205-223.

Vogt C.-A., Marans R.-W. (2004). Natural Resources and Open Space in the Residential Decision Process: a study of recent movers to fringe counties in southeast Michigan. Landscape and Urban Planning, 69, p. 255-269.

Ythier B. (1995). Espace rural, paysage et patrimoine. In «Paysage au pluriel, pour une approche ethnologique des paysages », Paris, Éditions de la Maison des sciences de l'homme, collection Ethnologie de la France, p. 185-193. 